\title{
Large reversible upconversion luminescence modification and 3D optical information storage in femtosecond laser irradiation-subjected photochromic glass
}

\author{
Daiwen $\mathrm{Xiao}^{1 \dagger}$, Xiongjian Huang ${ }^{2 \dagger}$, Yangke $\mathrm{Cun}^{1}$, Zhen $\mathrm{Hu}^{1}$, Zan $\mathrm{Xu}^{1}$, Xue Bai ${ }^{1}$, Yingzhu Zi ${ }^{1}$, Lixiang $\mathrm{Fu}^{1}$, \\ Asif Ali Haider ${ }^{1}$, Jianbei Qiu ${ }^{1}$, Zhiguo Song ${ }^{1}$, Guoping Dong ${ }^{2^{*}}$ and Zhengwen Yang ${ }^{1^{*}}$
}

\begin{abstract}
Fast response, high luminescence contrast, three-dimensional (3D) storage, and nondestructive reading are key factors for the optical storage application of photochromic materials. Femtosecond (fs) laser direct writing technology with multiphoton nonlinear absorption is becoming a useful tool for microprocessing functional units in the 3D space of glass owing to its remarkable advantages, such as a fast processing speed and high processing accuracy. Herein, the photochromism of transparent glass codoped with rare-earth ions was investigated under $800-\mathrm{nm}$ fs laser irradiation, affording a fast response. The photochromic glass achieves an upconversion luminescence (UCL) modification of 92\%. The photochromic glass can be bleached back to its original color using heat treatment. The transmittance and UCL modification show excellent reproducibility under alternating stimulations between 800-nm fs laser irradiation and heat treatment. The data can be written in the interior of the transparent photochromic glass using 800-nm fs laser irradiation, facilitating 3D information storage. These results suggest that the 800-nm fs laser irradiation-subjected photochromic glass is an ideal optical data storage medium.
\end{abstract}

Keywords: three-dimensional optical storage, glass, upconversion luminescence, reversible modification, nondestructive reading

\section{INTRODUCTION}

With the rapid increase in digitalized information, digitalized information storage in a reliable, safe, long-life, and low-cost manner is essential. Replacing the traditional magnetic data storage technology, the current optical storage technology with low energy consumption, low cost, and high storage capacity has attracted considerable attention [1-3]. However, the latter experiences a challenge in storage capacity improvement because of the optical diffraction limitation $[4,5]$. Therefore, the exploration of novel optical information storage media and technology is important [6-8]. Photochromic materials with absorption and photoluminescence modulation were recently developed as novel optical data storage media [9-11]. As established guiding rules for the optical storage application of photochromic materials, a fast photoresponse, large storage capacity, nondestructive reading, and high coloration and luminescence contrast are required [12,13]. Inorganic photochromic materials have garnered extensive attention as optical information storage media owing to their high strength or mechanical resistance, good thermal and chemical stabilities, and low environmental toxicity [14-16]. At present, many inorganic photochromic ceramics, such as $\mathrm{BaMgSiO}_{4}, \mathrm{Na}_{0.5} \mathrm{Bi}_{4.5^{-}}$ $\mathrm{Ti}_{4} \mathrm{O}_{15}$, and $\mathrm{PbWO}_{4}$, have been developed as optical data storage media, achieving the maximum downshift luminescence contrast of 96.3\% [12,17-27]. However, the three-dimensional (3D) storage of optical information cannot be realized using photochromic ceramics, limiting their storage capacity. Unlike opaque bulk ceramics, 3D optical storage can be relatively easily realized using transparent bulk glass [28]. Photochromic glass doped with rare-earth ions was recently developed. The photochromic glass obtained under 473-nm laser irradiation for $10 \mathrm{~min}$ exhibited a downshift luminescence modulation of $68 \%$, lower than that achieved by photochromic ceramics (96.3\%) [29]. Thus, the low luminescence contrast and long response time of photochromic glass have restricted its applications. Therefore, developing a novel transparent photochromic glass with fast response and high coloration contrast and luminescence modification as the optical storage media is important.

Nondestructive reading capability is crucial for the successful application of optical storage devices [30,31]. An upconversion luminescence (UCL)-modified glass doped with rare-earth ions can convert two or more photons with low energy into one photon with high energy [32-35]. Compared with downshift luminescence, UCL with a large anti-Stokes shift overcomes overlapping shortcomings between the UCL and excitation spectra of rare-earth ions, achieving nondestructive reading, which was recently reported in photochromic ceramics based on the UCL modification [36]. However, reports on the reversible UCL modification and optical information storage application of transparent photochromic glass are unavailable. Recently, femtosecond (fs) laser is becoming a useful tool for constructing novel photonic devices, such as optical waveguides, photonic crystals, and microgratings, affording a fast processing speed

\footnotetext{
${ }^{1}$ College of Materials Science and Engineering, Kunming University of Science and Technology, Kunming 650093, China

${ }^{2}$ State Key Laboratory of Luminescent Materials and Devices, School of Materials Science and Engineering, South China University of Technology, Guangzhou 510640, China

† These authors contributed equally to this work.

* Corresponding authors (emails: yangzw@kmust.edu.cn (Yang Z); dgp@scut.edu.cn (Dong G))
} 
and high processing accuracy [37-40]. The precise machining and information writing in the $3 \mathrm{D}$ space of the microregion of transparent glass can be realized using the multiphoton nonlinear absorption of fs laser [37,41]. Herein, the photochromism of glass doped with rare-earth ions was investigated using the $800-\mathrm{nm}$ fs laser direct writing technology, exhibiting fast and excellent photochromism. A photochromism reaction induced by the high UCL modification (92\%), which is higher than the maximum UCL modification reported in the case of photochromic ceramics [42], is observed in the photochromic glass. Optical nondestructive reading is achieved based on the UCL modification under 980-nm laser excitation. The data can be written in the interior of a transparent glass using $800-\mathrm{nm}$ fs laser irradiation, facilitating $3 \mathrm{D}$ information storage. The result suggests that the photochromic glass with high UCL modification and nondestructive reading shows potential as an optical data storage medium.

\section{EXPERIMENTAL SECTION}

\section{Sample preparation}

Borophosphate tellurite tungstate glasses were fabricated using different composites: $25 \mathrm{~B}_{2} \mathrm{O}_{3}-25 \mathrm{NaH}_{2} \mathrm{PO}_{4}-10 \mathrm{TeO}_{2}-(35-x) \mathrm{WO}_{3}-$ $5 \mathrm{Na}_{2} \mathrm{CO}_{3}-x \mathrm{Sb}_{2} \mathrm{O}_{3} \quad(x=0,1$, and $2 \mathrm{~mol} \%), 25 \mathrm{~B}_{2} \mathrm{O}_{3}-(24.7-y)$ $\mathrm{NaH}_{2} \mathrm{PO}_{4}-10 \mathrm{TeO}_{2}-34 \mathrm{WO}_{3}-5 \mathrm{Na}_{2} \mathrm{CO}_{3}-1 \mathrm{Sb}_{2} \mathrm{O}_{3}-0.3 \mathrm{Yb}_{2} \mathrm{O}_{3}-y \mathrm{Er}_{2} \mathrm{O}_{3}(y$ $=0,0.05,0.1,0.15$, and $0.20 \mathrm{~mol} \%)$, and $25 \mathrm{~B}_{2} \mathrm{O}_{3}-(24.9-z)$ $\mathrm{NaH}_{2} \mathrm{PO}_{4}-10 \mathrm{TeO}_{2}-34 \mathrm{WO}_{3}-5 \mathrm{Na}_{2} \mathrm{CO}_{3}-1 \mathrm{Sb}_{2} \mathrm{O}_{3}-0.1 \mathrm{Er}_{2} \mathrm{O}_{3}-z \mathrm{Yb}_{2} \mathrm{O}_{3}(z$ $=0.1,0.2,0.3,0.4$, and $0.50 \mathrm{~mol} \%)$. For this, stoichiometric raw materials $\left(\mathrm{B}_{2} \mathrm{O}_{3}, \mathrm{NaH}_{2} \mathrm{PO}_{4}, \mathrm{TeO}_{2}, \mathrm{WO}_{3}, \mathrm{Na}_{2} \mathrm{CO}_{3}, \mathrm{Yb}_{2} \mathrm{O}_{3}, \mathrm{Er}_{2} \mathrm{O}_{3}\right.$, and $\mathrm{Sb}_{2} \mathrm{O}_{3}$ ) were mixed homogeneously and melted in a furnace at $1050^{\circ} \mathrm{C}$ for $1 \mathrm{~h}$ in air. The melted solution was poured into a brass mold and cooled to room temperature (about $25^{\circ} \mathrm{C}$ ), affording borophosphate tellurite tungstate glasses codoped with various concentrations of $\mathrm{Yb}^{3+}$ and $\mathrm{Er}^{3+}$. The glasses were cut into a suitable size (length, width and height were 10,10, and $3 \mathrm{~mm}$, respectively) and polished after removing the internal stress using heat treatment. The photochromism of the borophosphate tellurite tungstate glasses was investigated under 800 -nm fs laser $\left(31 \mathrm{~W} \mathrm{~cm}^{-2}\right)$ irradiation for various times, and the spot size of the $800-\mathrm{nm}$ fs laser was $\sim 3 \mathrm{~mm}$. The photochromic glasses were bleached under thermal stimulation at different temperatures for various times.

\section{Sample characterization}

$\mathrm{X}$-ray diffraction (XRD) patterns of the borophosphate tellurite tungstate glasses before and after $800-\mathrm{nm}$ fs laser irradiation treatment were recorded on a D8 ADVANCE X-ray powder diffractometer (Bruker, Germany). The UCL spectra of the borophosphate tellurite tungstate glasses were measured under 980-nm laser excitation (HITACHIU-F-7000). The transmission spectra of the borophosphate tellurite tungstate glasses before and after $800-\mathrm{nm}$ fs laser irradiation treatment were measured on a U-4100 spectrophotometer. The electron paramagnetic resonance (EPR) spectra of the raw, photochromic, and bleached glasses were recorded at room temperature. The elemental distribution of the glasses before and after $800-\mathrm{nm}$ fs laser irradiation treatment was observed using scanning electron microscopy with energy-dispersive X-ray spectroscopy (EDS). The valence states of $\mathrm{W}$ and $\mathrm{Sb}$ in the raw, photochromic, and bleaching glasses were analyzed using X-ray photoelectron spectroscopy (XPS). The fs laser system, which shows no specific type, was provided by Spectra-Physics. The system comprised a seed light and an amplification stage. The pulse energy, frequency, and length of the fs laser were $0.02 \mathrm{~J}, 1000 \mathrm{~Hz}$, and $37 \mathrm{fs}$, respectively. The optical microscopic images of the glasses were captured using a (Nikon AZ100M) optical microscope equipped with a camera. The photochromic and UCL images were obtained using a complementary metal-oxide-semiconductor (CMOS) sensor camera (Nikon D7100, Japan) under daylight and $980-\mathrm{nm}$ laser excitation, respectively.

\section{RESULTS AND DISCUSSION}

The as-prepared $25 \mathrm{~B}_{2} \mathrm{O}_{3}-24.6 \mathrm{NaH}_{2} \mathrm{PO}_{4}-10 \mathrm{TeO}_{2}-(35-x) \mathrm{WO}_{3}-$ $5 \mathrm{Na}_{2} \mathrm{CO}_{3}-x \mathrm{Sb}_{2} \mathrm{O}_{3}-0.3 \mathrm{Yb}_{2} \mathrm{O}_{3}-0.1 \mathrm{Er}_{2} \mathrm{O}_{3}(x=0,1$, and $2 \mathrm{~mol} \%)$ glasses were termed WBP-Sb0, WBP-Sb1, and WBP-Sb2. Fig. S1a presents the images and transmission spectra of the WBP-Sb0, WBP-Sb1, and WBP-Sb2 glasses, showing that the transparency of the glasses was associated with the addition of $\mathrm{Sb}_{2} \mathrm{O}_{3}$. Compared with the WBP-Sb1 and WBP-Sb2 glasses, the WBP-Sb0 glass without $\mathrm{Sb}_{2} \mathrm{O}_{3}$ exhibited relatively low transmittance. The transmittance of WBP-Sb1 and WBP-Sb2 increased considerably after the addition of 1 or $2 \mathrm{~mol} \% \mathrm{Sb}_{2} \mathrm{O}_{3}$ in the glass composition, affording transparent glasses. The transmittance of the glasses was independent on the $\mathrm{Sb}_{2} \mathrm{O}_{3}$ concentration (Fig. S1a). Thus, the $\mathrm{Sb}_{2} \mathrm{O}_{3}$ concentration of $1 \mathrm{~mol} \%$ was selected for subsequent analysis. Fig. S1b, c show the XPS spectra of W in WBP-Sb0 and WBP-Sb1, respectively. The XPS image of WBP-Sb0 revealed two peaks (35.9 and $37.9 \mathrm{eV}$ ) of $\mathrm{W}^{6+}$ and two peaks ( 34.8 and $36.8 \mathrm{eV}$ ) of $\mathrm{W}^{5+}$. Notably, the peaks $(34.8$ and $36.7 \mathrm{eV})$ of $\mathrm{W}^{5+}$ in the XPS image of WBP-Sb1 decreased considerably after the addition of $\mathrm{Sb}_{2} \mathrm{O}_{3}$ (Fig. $\mathrm{S} 1 \mathrm{~b}, \mathrm{c}$ ). The polaron hopping between $\mathrm{W}^{6+}$ and $\mathrm{W}^{5+}$ afforded the coloration of WBP-Sb0 [43]. The addition of $\mathrm{Sb}_{2} \mathrm{O}_{3}$ in WBP-Sb1 limited the conversion from $\mathrm{W}^{6+}$ to $\mathrm{W}^{5+}$. Thus, the transparent WBP-Sb1 glass was obtained. Glasses codoped with various concentrations of $\mathrm{Yb}^{3+}$ and $\mathrm{Er}^{3+}$ were prepared. The influence of $\mathrm{Yb}^{3+}$ or $\mathrm{Er}^{3+}$ concentrations on the UCL intensity and transparency of glass was also investigated. All glasses exhibited a green UCL at 524 and $548 \mathrm{~nm}$ and a red UCL at $658 \mathrm{~nm}$ under 980-nm laser excitation (Fig. S2a-d). The optimal concentrations of $\mathrm{Er}^{3+}$ and $\mathrm{Yb}^{3+}$ in the glass were approximately 0.1 and $0.3 \mathrm{~mol} \%$, respectively. The logarithmic relation between the $980-\mathrm{nm}$ laser power and the UCL intensity of WBP-Sb1 (Fig. S3a) showed that the UCL at 524/548 and $658 \mathrm{~nm}$ corresponded to three- and two-photon processes, respectively, under 980-nm laser excitation; the possible UCL mechanism is proposed in Fig. S3b.

The photochromism of WBP-Sb1 was realized using $800-\mathrm{nm}$ fs laser direct writing technology (Fig. 1a). The fixed 800-nm fs laser was focused on the glass sample, and the glass could be moved by controlling the optical microscope platform. The $2 \mathrm{D}$ and $3 \mathrm{D}$ photochromic patterns can be written on the surface and in the interior of the glasses, respectively (Fig. 1a). Fig. 1b shows the transmission spectra and images of WBP-Sb1 subjected to $800-\mathrm{nm}$ fs laser $\left(31 \mathrm{~W} \mathrm{~cm}^{-2}\right.$, spot size $\left.3 \mathrm{~mm}\right)$ irradiation for various times. The transmittance and coloration of WBP-Sb1 changed after the glass was subjected to $800-\mathrm{nm}$ fs laser irradiation. The transmittance of WBP-Sb1 decreased with an increase in the $800-\mathrm{nm}$ fs laser irradiation time, and the glass became dark blue. The transmittance and coloration of the glass stabilized when the $800-\mathrm{nm}$ fs laser irradiation time exceeded $15 \mathrm{~s}$. Thus, the appropriate $800-\mathrm{nm}$ fs laser irradiation time was 
a

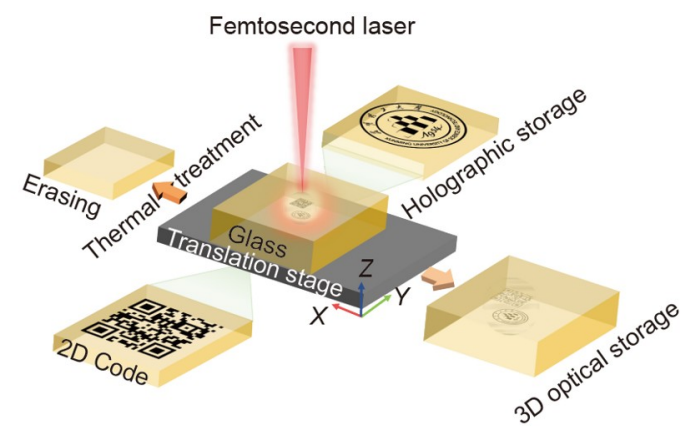

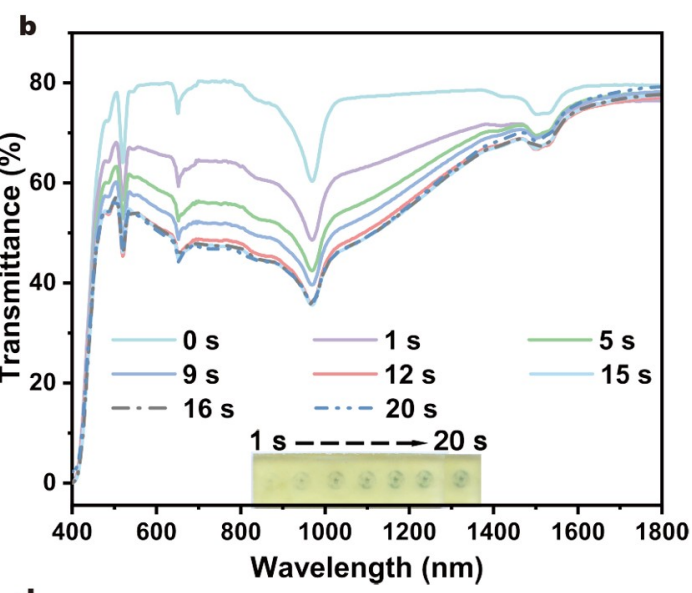

d

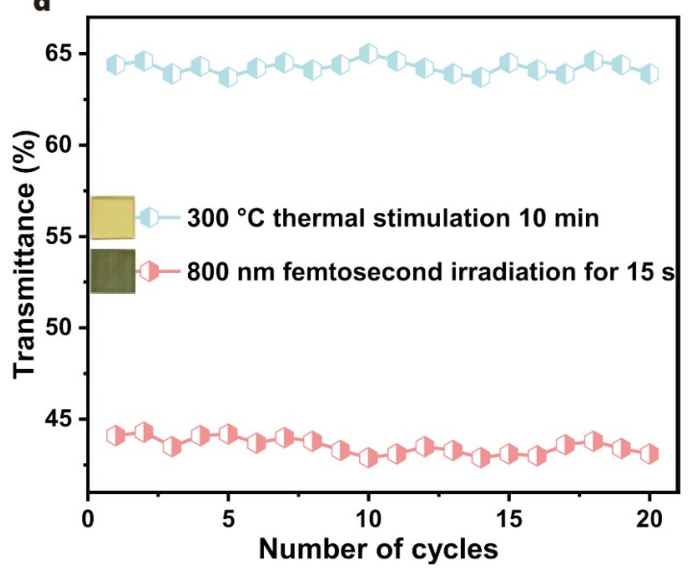

Figure 1 (a) Schematic of the writing and erasing of optical information. 2D and 3D photochromic patterns are recorded on the surface and in the interior of the glass, respectively, and the photochromic patterns can be erased using heat treatment. (b) Transmission spectra and images of the WBP-Sb1 glass subjected to $800-\mathrm{nm}$ fs laser irradiation for various times. (c) Transmission spectra of the photochromic WBP-Sb1 glass subjected to 800-nm fs laser irradiation after thermal stimulation for $10 \mathrm{~min}$ at various temperatures. (d) The 548-nm transmittance of the WBP-Sb1 glass under alternating stimulations between $800-\mathrm{nm}$ fs laser irradiation and heat treatment $\left(300^{\circ} \mathrm{C}, 10 \mathrm{~min}\right)$ as a function of the number of cycles.

set as $15 \mathrm{~s}$. The transmittance contrast $(\Delta R)$ of WBP-Sb1 was calculated using the equation $\Delta R=R_{0}-R_{1}$, where $R_{0}$ and $R_{1}$ represent the transmittance before and after $800-\mathrm{nm}$ fs laser irradiation, respectively. Fig. S4 shows that the 400-1600-nm transmission of WBP-Sb1 was modulated and the maximum $\Delta R$ value was $\sim 33 \%$ after $800-\mathrm{nm}$ fs laser irradiation for $15 \mathrm{~s}$. These results demonstrate that a photochromic glass with a fast response was obtained under $800-\mathrm{nm}$ fs laser irradiation, which is advantageous for optical storage applications.

The bleaching of the photochromic WBP-Sb1 glass induced by $800-\mathrm{nm}$ fs laser irradiation was investigated under thermal stimulation at various temperatures for $10 \mathrm{~min}$ (Fig. 1c). The transmittance of WBP-Sb1 increased with the stimulation temperature. The transmittance intensity of this glass returned to its original state when the stimulation temperature reached $300^{\circ} \mathrm{C}$. Fig. S5 presents the transmission spectra of the photochromic WBP-Sb1 glass treated for various times at $200^{\circ} \mathrm{C}$. The transmittance of the photochromic WBP-Sb1 glass increased with the stimulation time and was restored to its primitive state when the stimulation time was $2 \mathrm{~h}$. Fig. $1 \mathrm{~d}$ shows the $548-\mathrm{nm}$ transmission of WBP-Sb1 achieved under alternating stimulations between $800-\mathrm{nm}$ fs laser irradiation and $300^{\circ} \mathrm{C}$ heat treatment $(10 \mathrm{~min})$ as a function of the number of cycles. The photochromic WBP-Sb1 glass showed excellent rewritability and erasability.
Currently, several models have been proposed to explain the photochromism mechanism of materials. Reports indicate that the photochromism of $\mathrm{WO}_{3}$ films is related to the formation of $\mathrm{H}_{x} \mathrm{~W}_{x}^{6+} \mathrm{W}_{1-x}^{5+} \mathrm{O}_{3}$ [44-46]. The polarons hopping between $\mathrm{W}^{6+}$ and $\mathrm{W}^{5+} / \mathrm{W}^{4+}$ afford the photochromism of $\mathrm{PbWO}_{4}$ ceramics. Fig. S6a presents the XRD patterns of WBP-Sb1 before and after $800-n m$ fs laser irradiation treatment. The XRD peaks were not detected, and the amorphous phase of WBP-Sb1 before and after $800-\mathrm{nm}$ fs laser irradiation treatment was observed. Fig. S6b shows the normalized Raman spectra of WBP-Sb1 before and after $800-\mathrm{nm}$ fs laser irradiation treatment induced by the 800 $\mathrm{nm}$ fs laser irradiation. The identical Raman spectra of WBP-Sb1 before and after $800-\mathrm{nm}$ fs laser irradiation treatment suggested that the WBP-Sb1 glass structure before and after $800-\mathrm{nm}$ fs laser irradiation treatment remained the same. Oxygen vacancy peaks were not detected in the room-temperature EPR spectra of the raw and photochromic WBP-Sb1 glasses (Fig. S6c). EDS mapping revealed the uniform distribution of all elements in WBP-Sb1 before and after $800-\mathrm{nm}$ fs laser irradiation treatment, and element migration was not observed (Figs S7 and S8). Thus, the $800-\mathrm{nm}$ fs laser irradiation-induced photochromism of WBP-Sb1 was not attributed to the glass crystallization, structural change, oxygen vacancy formation, and element migration [47]. The glass without the codoping of $\mathrm{Yb}^{3+}$ and $\mathrm{Er}^{3+}$ was 
subjected to $800-\mathrm{nm}$ fs laser irradiation for $15 \mathrm{~s}$, exhibiting the same transmittance, in contrast to the WBP-Sb1 glass codoped with $\mathrm{Yb}^{3+}$ and $\mathrm{Er}^{3+}$ (Fig. S9). The XPS spectra of $\mathrm{Yb}^{3+}$ and $\mathrm{Er}^{3+}$ in WPB-Sb1 before and after $800-\mathrm{nm}$ fs laser irradiation treatment showed no changes (Fig. S10). This result demonstrates that the photochromism of WBP-Sb1 was unrelated to codoping using $\mathrm{Yb}^{3+}$ and $\mathrm{Er}^{3+}$ after $800-\mathrm{nm}$ fs laser irradiation. Fig. 2a-d present the XPS spectra of W and Sb in WPB-Sb1 before and after 800$\mathrm{nm}$ fs laser irradiation treatment. Compared with WPB-Sb1 before photochromism (Fig. 2a), the peaks of $\mathrm{W}^{5+}$ located at 34.7 and $36.8 \mathrm{eV}$ increased after photochromism (Fig. 2c). Two peaks of $\mathrm{Sb}^{3+}$ were detected at 530.7 and $540.1 \mathrm{eV}$ in WBP-Sb1 before photochromism, and no XPS peak of $\mathrm{Sb}^{5+}$ was observed before photochromism (Fig. 2b). Two XPS peaks of $\mathrm{Sb}^{5+}$ were detected at 531.2 and $540.6 \mathrm{eV}$ after photochromism (Fig. 2d) [48]. The $800-n m$ fs laser irradiation with high energy may induce the ionization of $\mathrm{Sb}^{3+}$, resulting in the conversion of $\mathrm{Sb}^{3+}$ to $\mathrm{Sb}^{5+}$ and the production of free electrons [49]. Free electrons were captured by $\mathrm{W}^{6+}$ in the glass, yielding $\mathrm{W}^{5+}$. The electrons localized around $\mathrm{W}^{5+}$ could "facilitate the polarization of the surrounding lattice, forming small polarons". The light stimulation could afford the mutual transition of polarons between two or three types of ions with different valence states, inducing light absorption. The reactions $\mathrm{Sb}^{3+}+h v \rightarrow \mathrm{Sb}^{5+}+2 \mathrm{e}^{-}$and $2 \mathrm{~W}^{6+}+h v$ $+2 \mathrm{e}^{-} \rightarrow 2 \mathrm{~W}^{5+}$ may occur in the case of WBP-Sb1 after $800-\mathrm{nm}$ fs laser irradiation. The hopping of polarons between $\mathrm{W}^{6+}$ and
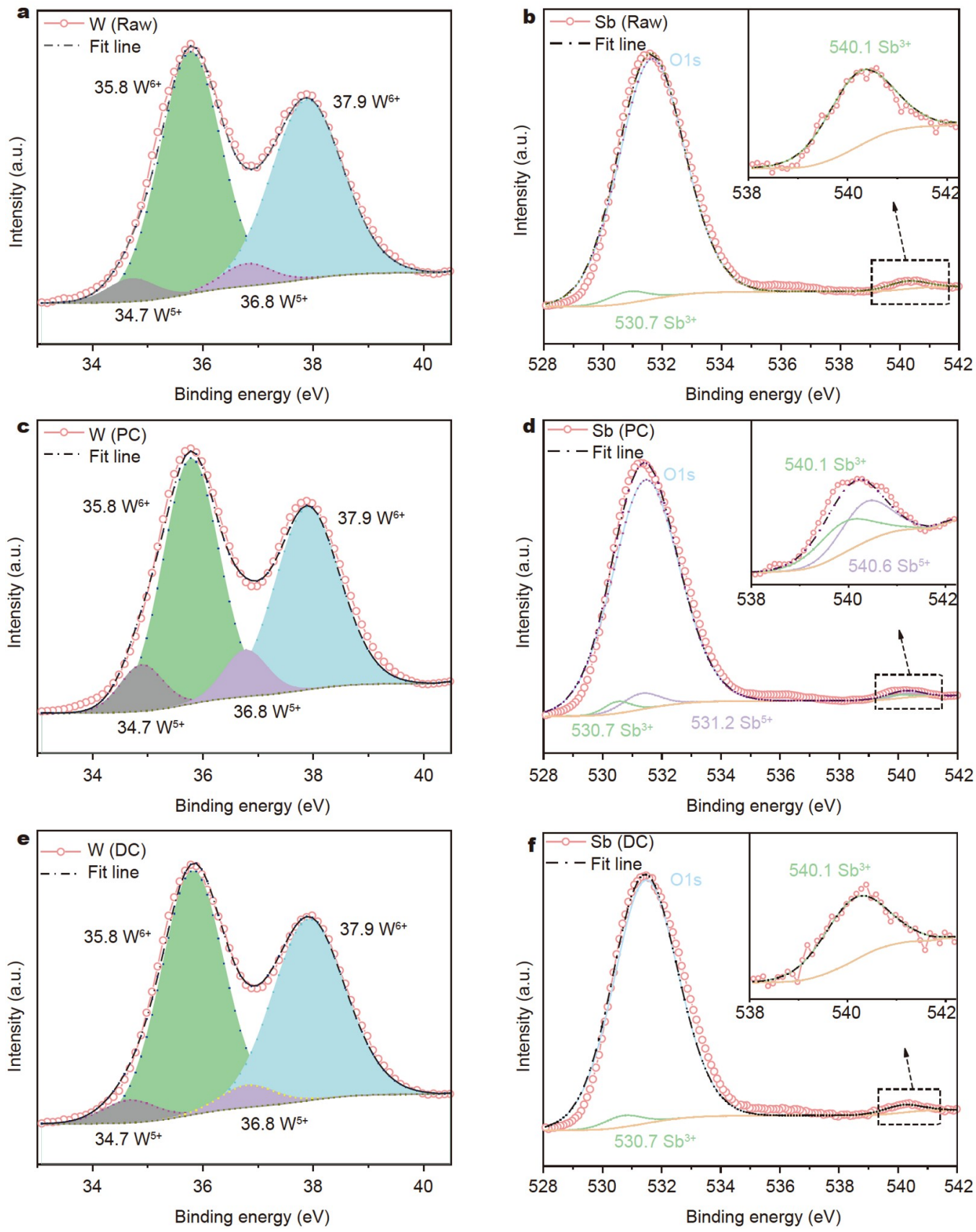

Figure 2 XPS spectra of (a) W and (b) Sb in the WBP-Sb1 glass before photochromism. XPS spectra of (c) W and (d) Sb in the photochromic (PC) WBP-Sb1 glass subjected to $800-\mathrm{nm}$ fs laser irradiation for $15 \mathrm{~s}$. XPS spectra of (e) W and (f) Sb in the decolored (DC) WBP-Sb1 glass stimulated at $300^{\circ} \mathrm{C}$ for 10 min. 
$\mathrm{W}^{5+}$ induced the photochromism of WBP-Sb1 [50-52].

The photochromic WBP-Sb1 glass subjected to $800-\mathrm{nm}$ fs laser irradiation for $15 \mathrm{~s}$ was bleached at $300^{\circ} \mathrm{C}$ for $10 \mathrm{~min}$. The XPS spectra of rare-earth ions and the XRD pattern, Raman spectra, and EPR spectra of the bleached WBP-Sb1 glass revealed that the glass structure, amorphous phase, oxygen vacancy, and valence state of rare-earth ions showed no change after thermal stimulation, contrary to the photochromic WBP-Sb1 glass (Figs S6 and S10). The XPS spectra of $\mathrm{W}$ and $\mathrm{Sb}$ in the bleached WPB-Sb1 glass $\left(300^{\circ} \mathrm{C}\right.$ for $\left.10 \mathrm{~min}\right)$ are presented in Fig. 2e, f, respectively. Compared with the photochromic WPB-Sb1 glass (Fig. 2c), the peaks of $\mathrm{W}^{5+}$ at 34.7 and $36.8 \mathrm{eV}$ decreased after thermal bleaching. The two XPS peaks of $\mathrm{Sb}^{5+}$ at 531.2 and $540.6 \mathrm{eV}$ disappeared. $\mathrm{W}^{5+}$ was oxidized to $\mathrm{W}^{6+}$ in air after thermal stimulation, and $\mathrm{Sb}^{5+}$ was reduced to $\mathrm{Sb}^{3+}$. In the case of the photochromic WBP-Sb1 glass, the reactions $2 \mathrm{~W}^{5+} \rightarrow 2 \mathrm{~W}^{6+}+$ $2 \mathrm{e}^{-}$and $\mathrm{Sb}^{5+}+2 \mathrm{e}^{-} \rightarrow \mathrm{Sb}^{3+}$ occurred after thermal stimulation. Thus, the thermal bleaching of the photochromic WPB-Sb1 glass was realized.

Photochromism and UCL were observed in the case of WBPSb1. The UCL of WBP-Sb1 can be modulated based on its photochromic reaction. Fig. 3a presents the UCL spectra of WBP-Sb1 subjected to $800-\mathrm{nm}$ fs laser irradiation for various times after $980-\mathrm{nm}$ laser excitation. The UCL intensity of the photochromic WBP-Sb1 glass decreased when the $800-\mathrm{nm}$ fs laser irradiation time was increased, exhibiting photochromisminduced UCL modification. The equation $\Delta R_{\mathrm{m}}=\left(R_{0}-R_{\mathrm{L}}\right) / R_{0} \times$ $100 \%$ was used to characterize the degree of UCL modulation, where $R_{0}$ and $R_{L}$ are the 548-nm UCL intensity of the as-prepared and photochromic glasses, respectively. As expected, the degree of UCL modulation increased with the $800-\mathrm{nm}$ fs laser irradiation time. The maximum UCL modification of WBP-Sb1 was $\sim 92 \%$ when the $800-\mathrm{nm}$ fs laser irradiation time reached $15 \mathrm{~s}$ (Fig. 3b). The highest degree of luminescence modification was $70 \%$ lower than that of the photochromic glass. The $92 \%$ UCL modification of the photochromic WBP-Sb1 glass after $800-\mathrm{nm}$ fs laser irradiation is higher than the maximum luminescence modification reported for the photochromic glass [29]. The photochromic WBP-Sb1 glass subjected to $800-\mathrm{nm}$ fs laser irradiation was bleached and exposed to heat treatment at various temperatures for $10 \mathrm{~min}$, and the corresponding UCL spectra are shown in Fig. 3c. The UCL intensity of the bleached WBP-Sb1 glass increased with the heat treatment temperature. The UCL recovery degree $\left(\Delta R_{\mathrm{r}}\right)$ was characterized using the equation $\Delta R_{\mathrm{r}}=R_{\mathrm{b}} / R_{0} \times 100 \%$, where $R_{0}$ and $R_{\mathrm{b}}$ are the $548-\mathrm{nm}$ UCL intensity of the as-prepared and decolored WBP-Sb1 glasses, respectively. The $\Delta R_{\mathrm{r}}$ value of the decolored WBP-Sb1 glass was $\sim 99 \%$ when the stimulation temperature reached $300^{\circ} \mathrm{C}$, and the UCL reversion of the decolored WBP-Sb1 glass was realized (Fig. $3 \mathrm{~d}$ ). The influence of the stimulation time at $200^{\circ} \mathrm{C}$ on the decoloration and UCL reversion of the photochromic glass were also observed (Fig. S11). The UCL of the photochromic WBP-Sb1 glass recovered to its initial state when the stimulation time was $2 \mathrm{~h}$. Fig. $3 \mathrm{e}$ presents the 548-nm UCL intensity of WBP-Sb1 under alternating stimulations between $800-\mathrm{nm}$ fs laser irradiation and $300^{\circ} \mathrm{C}$ heat treatment for $10 \mathrm{~min}$ after 980-nm laser excitation, showing no UCL deterioration. The reversible UCL modification of WBP-Sb1 was also realized. These results suggest that the photochromic glass with a high UCL modification shows potential applications as optical data storage media.

The UCL decay lifetime of WBP-Sb1 before and after $800-\mathrm{nm}$
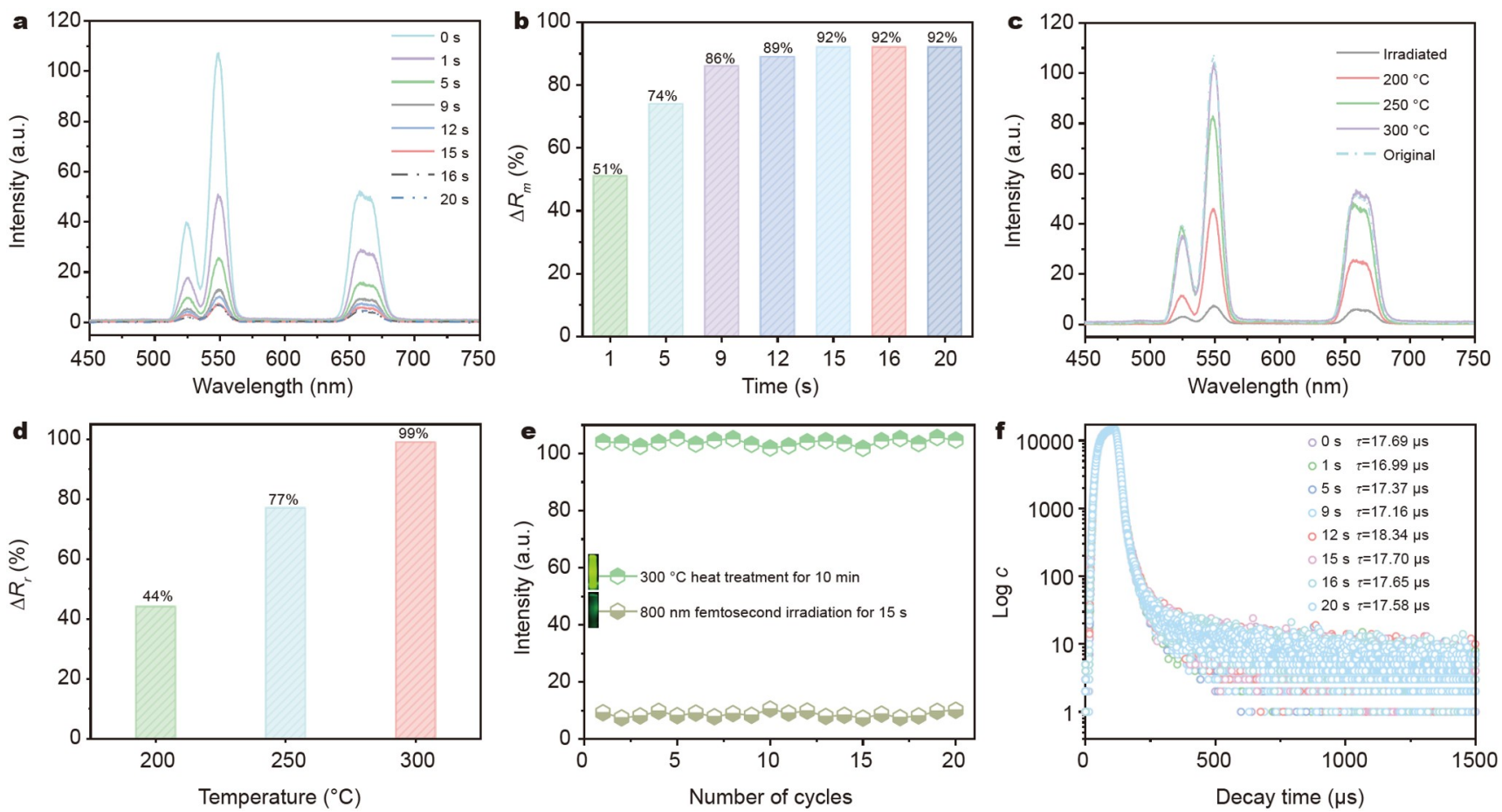

Figure 3 (a) UCL spectra and (b) degree of UCL modulation ( $\left.\Delta R_{\mathrm{m}}\right)$ of the WBP-Sb1 glass subjected to 800-nm fs laser irradiation for various times after 980$\mathrm{nm}$ laser excitation. (c) UCL spectra and (d) recovery degree $\left(\Delta R_{\mathrm{r}}\right)$ of the photochromic WBP-Sb1 glass subjected to heat treatment at various temperatures for $10 \mathrm{~min}$. (e) The 548-nm UCL intensity of WBP-Sb1 under alternating stimulations between 800 -nm fs laser irradiation and $300^{\circ} \mathrm{C} \mathrm{heat} \mathrm{treatment} \mathrm{(10} \mathrm{min)} \mathrm{as} \mathrm{a}$ function of the number of cycles. (f) Decay lifetime curves of the 548-nm intensity of the WBP-Sb1 glass subjected to 800 -nm fs laser irradiation for various times. 
fs laser irradiation treatment was measured to characterize the UCL modification mechanism. The UCL decay lifetime of the photochromic WBP-Sb1 glass subjected to $800-\mathrm{nm}$ fs laser irradiation for various times showed no changes compared with that of the raw WBP-Sb1 glass (Fig. 3f).

The results demonstrate that the UCL modification of WBP$\mathrm{Sb} 1$ was not attributed to the resonance energy transfer from $\mathrm{Er}^{3+}$ to the glass host. The absorption of the photochromic WBPSb1 glass overlapped with the UCL peaks of $\mathrm{Er}^{3+}$. The UCL modification was ascribed to the absorption of the photochromic WBP-Sb1 glass host.

Simple optical information patterns and a complex 2D code can be written on the WBP-Sb1 glass surface using the $800-\mathrm{nm}$ fs laser direct writing technology (Fig. 4a). The information "Kunming University of Science and Technology Official Website" (KUST) could be read out using a smartphone to scan the image of the $2 \mathrm{D}$ code. The information readout by scanning the $2 \mathrm{D}$ code using a smartphone at any time is consistent with the website content, realizing real-time updates of information (Supplementary Video 1). The encoded binary arrays corresponding to the "KUST" alphabets were written on the WBPSb1 glass surface using $800-\mathrm{nm}$ fs laser irradiation. Moreover, the encoded binary arrays corresponding to the "KUST" alphabets could be rewritten on the WBP-Sb1 glass surface using $800-\mathrm{nm}$ fs laser irradiation after erasing the encoded binary arrays under $300^{\circ} \mathrm{C}$ thermal stimulation for $10 \mathrm{~min}$ (Fig. $4 \mathrm{~b}$ ). The UCL pattern on the WBP-Sbl glass surface could also be realized after $980-\mathrm{nm}$ laser excitation based on the UCL modification induced by the photochromic reaction (Fig. 4c). The photochromic dot arrays were patterned on the WBP-Sb1 glass surface, which was subjected to $800-\mathrm{nm}$ fs laser irradiation for $15 \mathrm{~s}$. Weak UCL spectra of the photochromic dots were detected after the 980-nm laser excitation, and intense UCL spectra were observed in other regions without photochromism. The UCL modification spectra corresponded to the binary states of " 0 " and " 1 " (Fig. 4 d), realizing the nondestructive reading of bit-bybit UCL signals. Furthermore, the photochromism patterns and UCL modification were observed in the case of the glass codoped with $\mathrm{Yb}^{3+}$ and $\mathrm{Tb}^{3+}$ after the $800-\mathrm{nm}$ fs laser irradiation (Fig. S12), which are independent of the doping species of rareearth ions. This result suggests that the photochromic WBP-Sb1 glass doped with rare-earth ions is an ideal optical storage medium.

Unlike photochromic films and ceramics, the data could be written in the interior of the transparent WBP-Sb1 glass using the $800-\mathrm{nm}$ fs laser irradiation. Three rectangles with different sizes and Chinese characters "three", "four", and "five" were written in the interior of various layers of the glass by the focused $800-\mathrm{nm}$ fs laser ( $143 \mathrm{~kW} \mathrm{~cm}^{-2}$, spot size $\left.3 \mu \mathrm{m}\right)$ using the direct writing technology, which could be detected by employing an optical microscope (Fig. 5a). The Chinese characters in the interior of various layers of the glass were clearly and hierarchically read out using an optical microscope (Supplementary Video 2). The entire 3D pattern could be microprocessed in the glass interior using the $800-\mathrm{nm}$ fs laser irradiation (Fig. 5b), affording $3 \mathrm{D}$ information storage.

Other continuous lasers, such as those at $808\left(78 \mathrm{~W} \mathrm{~cm}^{-2}\right)$, $690\left(42 \mathrm{~W} \mathrm{~cm}^{-2}\right), 532\left(284 \mathrm{~W} \mathrm{~cm}^{-2}\right)$, or $473 \mathrm{~nm}\left(151 \mathrm{~W} \mathrm{~cm}^{-2}\right)$, were used to irradiate WBP-Sb1. No photochromism of WBPSb1 was observed under $808-\mathrm{nm}\left(78 \mathrm{~W} \mathrm{~cm}^{-2}\right)$, 690-nm $\left(42 \mathrm{~W} \mathrm{~cm}^{-2}\right)$, or $532-\mathrm{nm}\left(284 \mathrm{~W} \mathrm{~cm}^{-2}\right)$ continuous laser irradiation for $15 \mathrm{~min}$ (Fig. S13). However, WBP-Sb1 showed photochromism under $473-\mathrm{nm}\left(151 \mathrm{~W} \mathrm{~cm}^{-2}\right)$ continuous laser irradiation for $15 \mathrm{~min}$, and the photochromism reached

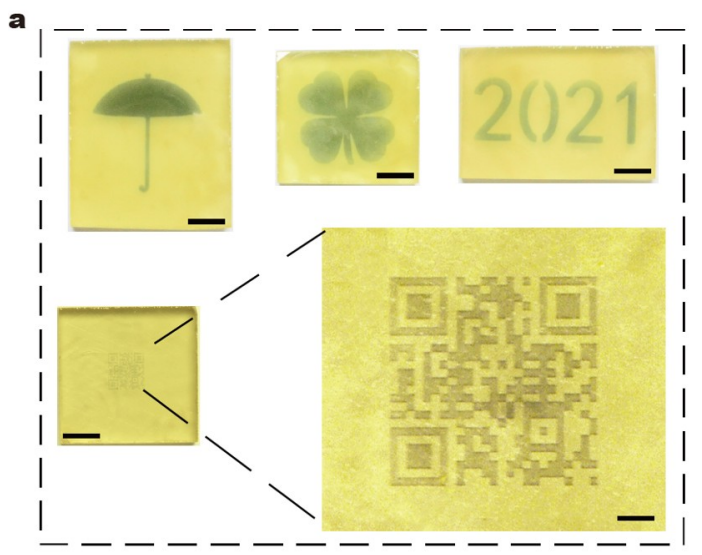

b
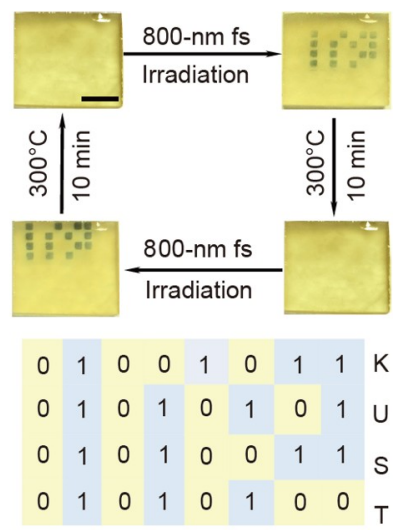

c

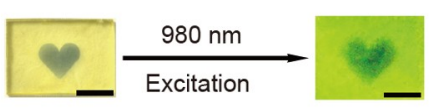

d

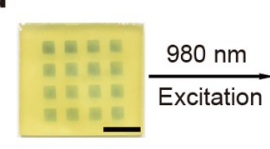

$\begin{array}{llllllllll}1 & 0 & 1 & 0 & 1 & 0 & 1 & 0 & 1 & 0\end{array}$

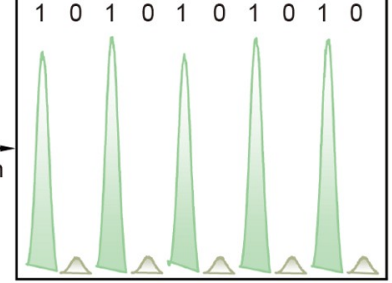

Figure 4 (a) Photochromic pattern information, digital information, and raw and enlarged photochromic 2D code pattern. (b) Encoded binary arrays recorded using $800-\mathrm{nm}$ fs laser; binary arrays erased by $300^{\circ} \mathrm{C}$ heat treatment for $10 \mathrm{~min}$, and corresponding binary code rewritten using $800-\mathrm{nm}$ fs laser. (c) Photochromic and UCL patterns after 980-nm laser excitation. (d) Photochromic dot arrays patterned on the WBP-Sb1 glass surface and the 548-nm UCL spectra of the corresponding photochromic dot arrays after 980-nm laser excitation; the weak UCL spectrum is from the photochromic dots, and the intense UCL spectrum is from the other region without photochromism. Scale bar: $5 \mathrm{~mm}$. 


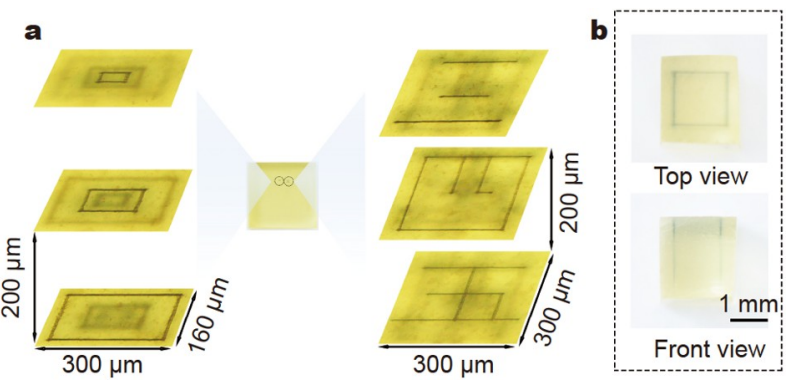

Figure 5 (a) Optical microscopic images of three rectangles with different sizes and Chinese characters "three", "four", and "five" recorded in various layers of the glass; (b) 3D pattern in the interior of the transparent glass from top and front views in daylight.

saturation when the 473-nm continuous laser irradiation time reached $15 \mathrm{~min}$ (Fig. S14a). The maximum $\Delta R$ value was $\sim 9.5 \%$ (Fig. S14b), lower than that obtained using the $800-\mathrm{nm}$ fs laser irradiation (33\%). Fig. S15a, b present the UCL spectra and degree of UCL modulation of the photochromic WBP-Sb1 glass subjected to $473-\mathrm{nm}$ continuous laser irradiation. The maximum UCL modification of WBP-Sb1 was $68 \%$ under the $473-\mathrm{nm}$ continuous laser irradiation time of $15 \mathrm{~min}$, lower than that obtained under the $800-\mathrm{nm}$ fs irradiation time of $15 \mathrm{~s}$ (92\%). Thus, these results demonstrate that a photochromic glass with high coloration contrast, good luminescence modification, and fast response was obtained under $800-\mathrm{nm}$ fs laser irradiation, which is advantageous for optical storage applications.

\section{CONCLUSIONS}

Overall, the fast response and excellent photochromism of the UCL glass doped with rare-earth ions were realized using the $800-n m$ fs laser direct writing technology. The photochromic glass exhibited excellent rewritability and erasability under alternating stimulations between $800-\mathrm{nm}$ fs irradiation and heat treatment. The maximum UCL modification (92\%) of the glass doped with rare-earth ions was achieved after 980-nm laser excitation based on the photochromism reaction. The $2 \mathrm{D}$ code was designed and recorded on the glass surface using the 800$\mathrm{nm}$ fs laser direct writing technology, realizing the real-time update of information by scanning the $2 \mathrm{D}$ code using a smartphone. The data could be written in the interior of the transparent glass using the $800-\mathrm{nm}$ fs laser irradiation, facilitating 3D information storage. The results suggest that the photochromic glass with high UCL modification and nondestructive reading shows potential applications in the fields of high-capacity optical data storage and information security.

\section{Received 21 October 2021; accepted 14 December 2021;} published online 20 January 2022

1 Gu M, Zhang Q, Lamon S. Nanomaterials for optical data storage. Nat Rev Mater, 2016, 1: 16070

2 Zhuang Y, Wang L, Lv Y, et al. Optical data storage and multicolor emission readout on flexible films using deep-trap persistent luminescence materials. Adv Funct Mater, 2018, 28: 1705769

3 Gu M, Li X, Cao Y. Optical storage arrays: A perspective for future big data storage. Light Sci Appl, 2014, 3: e177

4 Trelles O, Prins P, Snir M, et al. Big data, but are we ready? Nat Rev Genet, 2011, 12: 224

5 Zijlstra P, Chon JWM, Gu M. Five-dimensional optical recording mediated by surface plasmons in gold nanorods. Nature, 2009, 459:
410-413

6 Zhang J, Gecevičius M, Beresna M, et al. Seemingly unlimited lifetime data storage in nanostructured glass. Phys Rev Lett, 2014, 112: 033901

7 Lin S, Lin H, Ma C, et al. High-security-level multi-dimensional optical storage medium: Nanostructured glass embedded with $\mathrm{LiGa}_{5} \mathrm{O}_{8}: \mathrm{Mn}^{2+}$ with photostimulated luminescence. Light Sci Appl, 2020, 9: 22

8 Zhuang $\mathrm{Y}$, Chen $\mathrm{D}$, Chen $\mathrm{W}$, et al. X-ray-charged bright persistent luminescence in $\mathrm{NaYF}_{4}: \mathrm{Ln}^{3+} @ \mathrm{NaYF}_{4}$ nanoparticles for multidimensional optical information storage. Light Sci Appl, 2021, 10: 132

9 Zhang Z, Guo L, Sun H, et al. Rare earth orthoniobate photochromics with self-activated upconversion emissions for high-performance optical storage applications. J Mater Chem C, 2021, 9: 13841-13850

10 Yang Z, Hu J, Martin LIDJ, et al. Realizing nondestructive luminescence readout in photochromic ceramics via deep ultraviolet excitation for optical information storage. J Mater Chem C, 2021, 9: 14012-14020

11 Zhang Y, Luo L, Li K, et al. Reversible up-conversion luminescence modulation based on UV-VIS light-controlled photochromism in $\mathrm{Er}^{3+}$ doped $\mathrm{Sr}_{2} \mathrm{SnO}_{4}$. J Mater Chem C, 2018, 6: 13148-13156

12 Yang Z, Du J, Martin LIDJ, et al. Designing photochromic materials with large luminescence modulation and strong photochromic efficiency for dual-mode rewritable optical storage. Adv Opt Mater, 2021, 9: 2100669

13 Yang Z, Du J, Martin LIDJ, et al. Highly responsive photochromic ceramics for high-contrast rewritable information displays. Laser Photonics Rev, 2021, 15: 2000525

14 Bange K. Colouration of tungsten oxide films: A model for optically active coatings. Sol Energy Mater Sol Cells, 1999, 58: 1-131

15 He T, Yao J. Photochromism in composite and hybrid materials based on transition-metal oxides and polyoxometalates. Prog Mater Sci, 2006, 51: $810-879$

16 Wang S, Fan W, Liu Z, et al. Advances on tungsten oxide based photochromic materials: Strategies to improve their photochromic properties. J Mater Chem C, 2019, 7: 10119

17 Ren Y, Yang Z, Li M, et al. Reversible upconversion luminescence modification based on photochromism in $\mathrm{BaMgSiO}_{4}: \mathrm{Yb}^{3+}, \mathrm{Tb}^{3+}$ ceramics for anti-counterfeiting applications. Adv Opt Mater, 2019, 7: 1900213

18 Zhu Y, Sun H, Jia Q, et al. Site-selective occupancy of $\mathrm{Eu}^{2+}$ toward high luminescence switching contrast in $\mathrm{BaMgSiO}_{4}$-based photochromic materials. Adv Opt Mater, 2021, 9: 2001626

19 Ren Y, Yang Z, Wang Y, et al. Reversible multiplexing for optical information recording, erasing, and reading-out in photochromic $\mathrm{BaMgSiO}_{4}: \mathrm{Bi}^{3+}$ luminescence ceramics. Sci China Mater, 2020, 63: 582592

20 Li K, Luo L, Zhang Y, et al. Tunable luminescence contrast in photochromic ceramics $(1-x) \mathrm{Na}_{0.5} \mathrm{Bi}_{0.5} \mathrm{TiO}_{3-x} \mathrm{Na}_{0.5} \mathrm{~K}_{0.5} \mathrm{NbO}_{3}: 0.002 \mathrm{Er}$ by an electric field poling. ACS Appl Mater Interfaces, 2018, 10: 41525-41534

21 Bai X, Yang Z, Zhan Y, et al. Novel strategy for designing photochromic ceramic: Reversible upconversion luminescence modification and optical information storage application in the $\mathrm{PbWO}_{4}: \mathrm{Yb}^{3+}, \mathrm{Er}^{3+}$ photochromic ceramic. ACS Appl Mater Interfaces, 2020, 12: 21936-21943

22 Lin J, Zhou Y, Lu Q, et al. Reversible modulation of photoenergy in Smdoped $\left(\mathrm{K}_{0.5} \mathrm{Na}_{0.5}\right) \mathrm{NbO}_{3}$ transparent ceramics via photochromic behavior. J Mater Chem A, 2019, 7: 19374-19384

23 Smith AT, Ding H, Gorski A, et al. Multi-color reversible photochromisms via tunable light-dependent responses. Matter, 2020, 2: 680-696

24 Ruan J, Yang Z, Wen Y, et al. Laser induced thermochromism and reversible upconversion emission modulation of a novel $\mathrm{WO}_{3}: \mathrm{Yb}^{3+}, \mathrm{Er}^{3+}$ ceramic: Dual-modal fingerprint acquisition application. Chem Eng J, 2020, 383: 123180

25 Lv Y, Zhang S, Li Z, et al. Reversible multiplexing optical information storage and photoluminescence switching in $\mathrm{Eu}^{2+}$-doped fluorophosphate-based tunable photochromic materials. J Mater Chem C, 2021, 9: 5930-5944

26 Wei T, Jia B, Shen L, et al. A new class of upconversion luminescence tuning materials based on non-photochromic reaction: $\mathrm{Er}^{3+}$-activated $\mathrm{Ba}_{0.7} \mathrm{Sr}_{0.3} \mathrm{Nb}_{2} \mathrm{O}_{6}$ ferroelectrics. Acta Mater, 2021, 205: 116557

27 Chai J, Shao Z, Wang $\mathrm{H}$, et al. Ultrafast processes in photochromic 
material $\mathrm{YH}_{x} \mathrm{O}_{y}$ studied by excited-state density functional theory simulation. Sci China Mater, 2020, 63: 1579-1587

28 Huang X, Guo Q, Kang S, et al. Three-dimensional laser-assisted patterning of blue-emissive metal halide perovskite nanocrystals inside a glass with switchable photoluminescence. ACS Nano, 2020, 14: 31503158

29 Arora S, Bauer T, Barczyk R, et al. Direct quantification of topological protection in symmetry-protected photonic edge states at telecom wavelengths. Light Sci Appl, 2021, 10: 9

30 Kärnbratt J, Hammarson M, Li S, et al. Photochromic supramolecular memory with nondestructive readout. Angew Chem Int Ed, 2010, 49: 1854-1857

31 Zhang C, Zhou HP, Liao LY, et al. Luminescence modulation of ordered upconversion nanopatterns by a photochromic diarylethene: Rewritable optical storage with nondestructive readout. Adv Mater, 2010, 22: 633-637

32 Haase M, Schäfer H. Upconverting nanoparticles. Angew Chem Int Ed, 2011, 50: 5808-5829

33 Wilhelm S. Perspectives for upconverting nanoparticles. ACS Nano, 2017, 11: 10644-10653

34 Liu Y, Lu Y, Yang X, et al. Amplified stimulated emission in upconversion nanoparticles for super-resolution nanoscopy. Nature, 2017, 543: 229-233

35 Yau Y, Zeighami Y, Baker TE, et al. Network connectivity determines cortical thinning in early Parkinson's disease progression. Nat Commun, 2018, 9: 12

36 Zhang Q, Yue S, Sun $\mathrm{H}$, et al. Nondestructive up-conversion readout in $\mathrm{Er} / \mathrm{Yb}$ co-doped $\mathrm{Na}_{0.5} \mathrm{Bi}_{2.5} \mathrm{Nb}_{2} \mathrm{O}_{9}$-based optical storage materials for optical data storage device applications. J Mater Chem C, 2017, 5: 38383847

37 Tan D, Sharafudeen KN, Yue Y, et al. Femtosecond laser induced phenomena in transparent solid materials: Fundamentals and applications. Prog Mater Sci, 2016, 76: 154-228

38 Porfirev AP, Ustinov AV, Khonina SN. Polarization conversion when focusing cylindrically polarized vortex beams. Sci Rep, 2016, 6: 6

39 Zhang B, Wang L, Chen F. Recent advances in femtosecond laser processing of $\mathrm{LiNbO}_{3}$ crystals for photonic applications. Laser Photonics Rev, 2020, 14: 1900407

40 Chai N, Liu Y, Yue Y, et al. 3D nonlinear photolithography of tin oxide ceramics via femtosecond laser. Sci China Mater, 2021, 64: 1477-1484

41 Huang X, Guo Q, Yang D, et al. Reversible 3D laser printing of perovskite quantum dots inside a transparent medium. Nat Photonics, 2019, 14: 82-88

42 Sun $\mathrm{H}, \mathrm{Li}$ X, Zhu Y, et al. Achieving multicolor emission readout and tunable photoswitching via multiplexing of dual lanthanides in ferroelectric oxides. J Mater Chem C, 2019, 7: 5782-5791

43 Chen $\mathrm{M}$, Lin X, Dinh TH, et al. Configurable phonon polaritons in twisted $\mathrm{a}-\mathrm{MoO}_{3}$. Nat Mater, 2020, 19: 1307-1311

$44 \mathrm{He} \mathrm{Y,} \mathrm{Wu} \mathrm{Z,} \mathrm{Fu} \mathrm{L,} \mathrm{et} \mathrm{al.} \mathrm{Photochromism} \mathrm{and} \mathrm{size} \mathrm{effect} \mathrm{of} \mathrm{WO}_{3}$ and $\mathrm{WO}_{3}-\mathrm{TiO}_{2}$ aqueous sol. Chem Mater, 2003, 15: 4039-4045

45 Dachraoui H, Rupp RA, Lengyel K, et al. Photochromism of doped terbium gallium garnet. Phys Rev B, 2006, 74: 144104

46 Baum F, Pretto T, Brolo AG, et al. Uncovering the mechanism for the formation of copper thioantimonate $\left(\mathrm{Sb}^{\mathrm{V}}\right)$ nanoparticles and its transition to thioantimonide (Sb ${ }^{\mathrm{III}}$ ). Cryst Growth Des, 2018, 18: 6521-6527

47 Sokolov AV, Walker DR, Yavuz DD, et al. Femtosecond light source for phase-controlled multiphoton ionization. Phys Rev Lett, 2001, 87: 033402

48 Liu B, Wang J, Wu J, et al. Proton exchange growth to mesoporous $\mathrm{WO}_{3} \cdot 0.33 \mathrm{H}_{2} \mathrm{O}$ structure with highly photochromic sensitivity. Mater Lett, 2013, 91: 334-337

49 He T, Yao J. Photochromism of molybdenum oxide. J Photochem Photobiol C-Photochem Rev, 2003, 4: 125-143

50 Poirier G, Nalin M, Cescato L, et al. Bulk photochromism in a tungstate-phosphate glass: A new optical memory material? J Chem Phys, 2006, 125: 161101

51 Poirier G, Nalin M, Messaddeq Y, et al. Photochromic properties of tungstate-based glasses. Solid State Ion, 2007, 178: 871-875

52 Poirier G, Messaddeq Y, Ribeiro SJL, et al. Structural study of tungstate fluorophosphate glasses by Raman and X-ray absorption spectroscopy. J Solid State Chem, 2005, 178: 1533-1538

Acknowledgements This work was supported by the National Natural Science Foundation of China (51762029), the Applied Basic Research Key Program of Yunnan Province (2018FA026), and the Key Project of the National Natural Science Foundation of China-Yunnan Joint Fund (U2102215).

Author contributions Yang Z and Dong G conceived, designed, and supervised the overall project. Xiao D and Huang X performed the experiments. All the authors discussed the results and commented on the manuscript.

Conflict of interest The authors declare that they have no conflict of interest.

Supplementary information Supporting data are available in the online version of the paper.

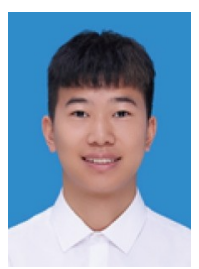

Daiwen Xiao is currently a graduate student at Kunming University of Science and Technology under the supervision of Prof. Zhengwen Yang. His current interest includes the fabrication of photochromic glass for optical storage applications.

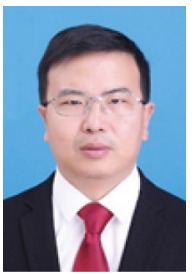

Zhengwen Yang is currently a professor at the College of Materials Science and Engineering, Kunming University of Science and Technology. He obtained his bachelor's degree in 2002 and master's degree in 2005 from Jilin University, respectively. He received his $\mathrm{PhD}$ degree from Tsinghua University in 2009. His research interests include the modification and enhancement of upconversion luminescence.

\section{飞秒激光辐照诱导光致变色玻璃中的上转换发光可 逆调控和3D光学信息存储}

肖代 文 $^{1 \dagger}$, 黄雄健 ${ }^{2 \dagger}$, 寸阳珂 ${ }^{1}$, 胡振 ${ }^{1}$, 徐赞 $^{1}$, 白雪 ${ }^{1}$, 字映竹 ${ }^{1}$, 付丽香 ${ }^{1}$, Asif Ali Haider ${ }^{1}$, 邱建备 ${ }^{1}$, 宋志国 ${ }^{1}$, 董国平 ${ }^{2 *}$, 杨正文 ${ }^{1 *}$

摘要 快速响应、高发光对比度、三维存储和信息无损读取是光致变 色材料光存储应用的几个关键因素. 具有多光子非线性吸收的飞秒激 光直写技术以其加工速度快、加工精度高等独特优势，正成为玻璃三 维空间微加工功能单元的有效工具. 本工作研究了 $800 \mathrm{~nm}$ 飞秒激光照 射下稀土离子掺杂透明玻璃的光致变色性质，结果证实在 $800 \mathrm{~nm}$ 飞秒 激光辐照下玻璃展现了快速光致变色响应. 在光致变色玻璃中实现了 $92 \%$ 的上转换发光调控. 光致变色玻璃可通过热刺激漂白回原色, 在 $800 \mathrm{~nm}$ 飞秒激光辐照和热处理交替刺激下，光致变色玻璃的透射率和 上转换调控表现出良好的重现性. 通过 $800 \mathrm{~nm}$ 飞秒激光照射, 可将数 据信息写入透明玻璃内部, 实现三维信息存储. 结果表明, $800 \mathrm{~nm}$ 飞秒 激光辐照诱导的光致变色玻璃是理想的光学数据存储介质. 